\title{
Quality Improvement: Undergraduate Contribution to Identify Barriers to Patient Discharge Timeliness
}

\author{
Kelsey Gatton ${ }^{1}$, Anamarie Black ${ }^{1}$, Preetham Talari MDํㅜ, Karen Clancy PhD ${ }^{1}$ \\ ${ }^{1}$ Clinical Leadership \& Management, College of Health Sciences, and Division of Hospital \\ Medicine, Department of Internal Medicine, University of Kentucky, Lexington, KY, USA
}

\section{ABSTRACT}

The undergraduate quality improvement project goal was to determine the primary barriers to timely discharges through a clinical leadership practicum experience at an advanced tertiary care teaching hospital. Delays in discharges are associated with adverse health outcomes and diminished efficiency within organizations. The authors utilized focused patient care provider interviews to identify barriers to timely discharges systematically. The study's metrics included comparisons of: 1) discharge orders placed before $10 \mathrm{a} . \mathrm{m}$. and 2) patients discharged before noon. The primary barriers found were ambulance transport delays and scheduled treatments after target discharge times. Early identification of patients planned to discharge aided in readiness and increased numbers of patients discharged before noon. The information collected by undergraduate students will help address the primary barriers and assist further quality improvement initiatives within the affinity group.

\section{Introduction}

Discharge efficiency impacts hospital performance. Delays following the issue of discharge orders contribute to increases in medical errors, longer lengths of stay, higher costs, and diminished patient satisfaction (Ragavan et al., 2017). A systematic literature review found that discharge delays are associated with adverse effects such as decreased mobility and increases in mortality, infection, and costs. (Rojas-Garcia et al., 2017) Furthermore, discharge delays negatively impact patient experience and health outcomes alongside staff morale and stress levels (Rojas-Garcia et al., 2017).

One observational study at a large academic medical center categorized barriers to timely discharge as diagnosis confirmation, test results or procedures, physician consultation, medication, and transportation (Rohatgi et al., 2018). Common themes creating delays in the discharge process are known to include communication, limited knowledge of roles, and scarce resources (Okoniewska et al., 2015).

This project was conducted at a large tertiary care teaching hospital that utilizes multidisciplinary affinity groups to achieve quality and process improvements. The Discharge Efficiency (DE) affinity group is charged to combat discharge delays and other quality improvement issues. Project investigators were undergraduate clinical leadership practicum students working with the DE affinity group during the fall semester of 2020. The student investigators demonstrated how to effectively apply qualitative data collection methods, critical thinking, data analysis and interpretation to quality improvement projects in an advanced clinical care setting.

\begin{abstract}
Aims
The undergraduate practicum quality improvement project goal was to determine the primary barriers to timely discharges at an advanced tertiary care teaching hospital. The secondary goal was to propose practical strategies to reduce the time between discharge order placement and final discharge completion to two hours.
\end{abstract}




\section{Methods}

\section{Sampling}

To identify discharge delays, this quality improvement project utilized data manually retrieved from Sunrise Clinical Manager (SCM) and hospital staff communication via Voalte. SCM is the electronic medical record system used at the practicum site. Voalte is a Health Insurance Portability and Accountability Act (HIPAA) compliant clinical communication software. From September 9 to November 6, 2020, de-identified data was pulled from SCM two to four times per week and compiled in a collaborative spreadsheet. The information collected included bed numbers, medicine team coverage, discharge order times, and final discharge times. A total of 165 patients were included in the project, with a final sample size of 64 . Of the 165,101 discharges were excluded due to "no staff response" or "successful discharge before noon."

Project metrics were; 1) discharge order placed before 10 a.m. and 2) final discharge completed by noon. Primary methods of the collection included qualitative interviews and subsequent coding of those interviews. The metrics were used based on data that had been previously collected and tools currently used by the organization. Specifically, the "M20 List" is a consulting tool that identifies patients who should be discharged by noon (DBN) the following day.

\section{Data Collection}

Data collection and identifying barriers to patient discharge occurred in two major groups. Patients identified to be discharged before noon the next day were part of the M20 specific group. The M20 list is used by case management, physicians, nurses, and other patient care staff. Placement on this list occurs by 4 p.m. after 2:30 p.m. team huddles the day before collection. After 4 p.m., students checked SCM and input the selected bed numbers in the spreadsheet. At 10 a.m. the next day, SCM was reviewed again to determine which beds had a discharge order before 10 a.m. If an M20 patient had an order placed by 10 a.m., the bedside nurse was contacted and interviewed via Voalte to determine any expected barriers. If an M20 patient did not have an order by 10 a.m., the case manager or social worker was interviewed to record why. At noon, patients who were still in SCM were recorded as not discharged by noon. Patient care staff were interviewed again to determine and record final barriers to discharge.

The second major group involved patients who were not identified for the M20 list but had an order by 10 a.m. Students interviewed bedside nurses via Voalte to determine if any discharge barriers were expected for these patients. SCM was again checked at noon to determine which beds had not been discharged by the noon metric. If beds were still in the system, students interviewed the same nurses to record the actual patient discharge barriers. Finally, the case manager or social worker for the bed was interviewed to inquire why they could not be identified for the M20 list the day before. Table 1 outlines the prompts utilized during Voalte communication.

Table 1. Voalte messaging prompts utilizing during staff communication

\begin{tabular}{|l|c|}
\hline 10 a.m. Bedside Nurse & $\begin{array}{c}\text { Good morning STAFF NAME, my name is } \\
\text { RESEARCHER NAME, and I am working with your } \\
\text { unit nurse manager and PRECEPTOR. We are working } \\
\text { on finding barriers to discharging patients before noon } \\
\text { if they have a discharge order before } 10 \text { a.m. Do you } \\
\text { see any barriers to discharge ROOM \# before noon as } \\
\text { this patient has an order before } 10 \text { a.m.? }\end{array}$ \\
\hline
\end{tabular}




\begin{tabular}{|c|c|}
\hline 10 a.m. Case Management (M20, with order) & $\begin{array}{l}\text { Good morning STAFF NAME, my name is } \\
\text { RESEARCHER NAME, and I am working with CASE } \\
\text { MANAGEMENT LEADER and PRECEPTOR. We are } \\
\text { working on our early discharge culture and are collect- } \\
\text { ing relevant data. ROOM \# is on the M } 20 \text { list and has } \\
\text { an order before } 10 \text { a.m. Do you see any barriers to dis- } \\
\text { charging them before noon as this patient has an order } \\
\text { before } 10 \text { a.m.? }\end{array}$ \\
\hline Noon Case Management (M20, without order) & $\begin{array}{l}\text { Good morning STAFF NAME, my name is } \\
\text { RESEARCHER NAME, and I am working with CASE } \\
\text { MANAGEMENT LEADER and PRECEPTOR. We are } \\
\text { working on our early discharge culture and are collect- } \\
\text { ing relevant data. ROOM \# is on the M20 list but did } \\
\text { not have an order before } 10 \text { a.m. Do you see any barri- } \\
\text { ers to discharging them before noon? }\end{array}$ \\
\hline 12 p.m. Case Management & $\begin{array}{l}\text { Good morning STAFF NAME, my name is } \\
\text { RESEARCHER NAME, and I am working with CASE } \\
\text { MANAGEMENT LEADER and PRECEPTOR. We are } \\
\text { working on our early discharge culture and are collect- } \\
\text { ing relevant data. ROOM \# was not on the M } 20 \text { list but } \\
\text { had a discharge order before } 10 \text { a.m. Would you know } \\
\text { why or if they could've been identified for the M20 list } \\
\text { yesterday? }\end{array}$ \\
\hline
\end{tabular}

\section{Analysis}

In the collaborative spreadsheet, data collected included the number of patients selected, patients with a pending order at 10 a.m., and patients discharged before noon for each major group. In a separate sheet, bed numbers, teams, staff contact names, 10 a.m. status, DBN status, and discharge barriers were recorded. Individual sheets were used to categorize and analyze barriers into specific groups. Barriers and delays were divided into eleven groups: patient decompensation, ambulance, family transport, pharmacy, education, treatment scheduled, nursing, MD, insurance, and placement. Data were summarized using counts and percentages. The analysis was performed through Google Sheets functions, including sums and averages.

\section{Results}

After conducting the data collection process, the investigators could differentiate the primary barriers to the patient discharge process. The major causes of diminished discharge timeliness were transportation delays, scheduled treatments, and overall lack of communication. The leading causes were ambulance delays and treatments scheduled after the target discharge time, specific to discharge barriers. Transport ambulances are often planned and rescheduled past noon due to availability. Patients needing chemotherapy, intravenous medications, radiology exams, or other treatments often received these after the noon metric. 
The most significant barrier was ambulance delay. Of the 64 categorizable responses, ambulance delays constituted 20 of those. The second largest barrier to timely discharge was having a treatment scheduled. There were 14 instances of this type of delay. There were 84 patients selected for the M20 list. Of these patients, 50 had a discharge order by 10 a.m., and 42 were DBN. Percentage-wise, $59.5 \%$ had an order before 10 a.m., and $84 \%$ of those were DBN. As for the second group, 116 patients were not on the M20 list but had an order by 10 a.m. Of these patients, 47 were DBN for a percentage of $40.5 \%$. Of all beds with an order before 10 a.m., $53.6 \%$ of those patients were DBN. Visual representation of the barrier occurrences is in Figure 1.

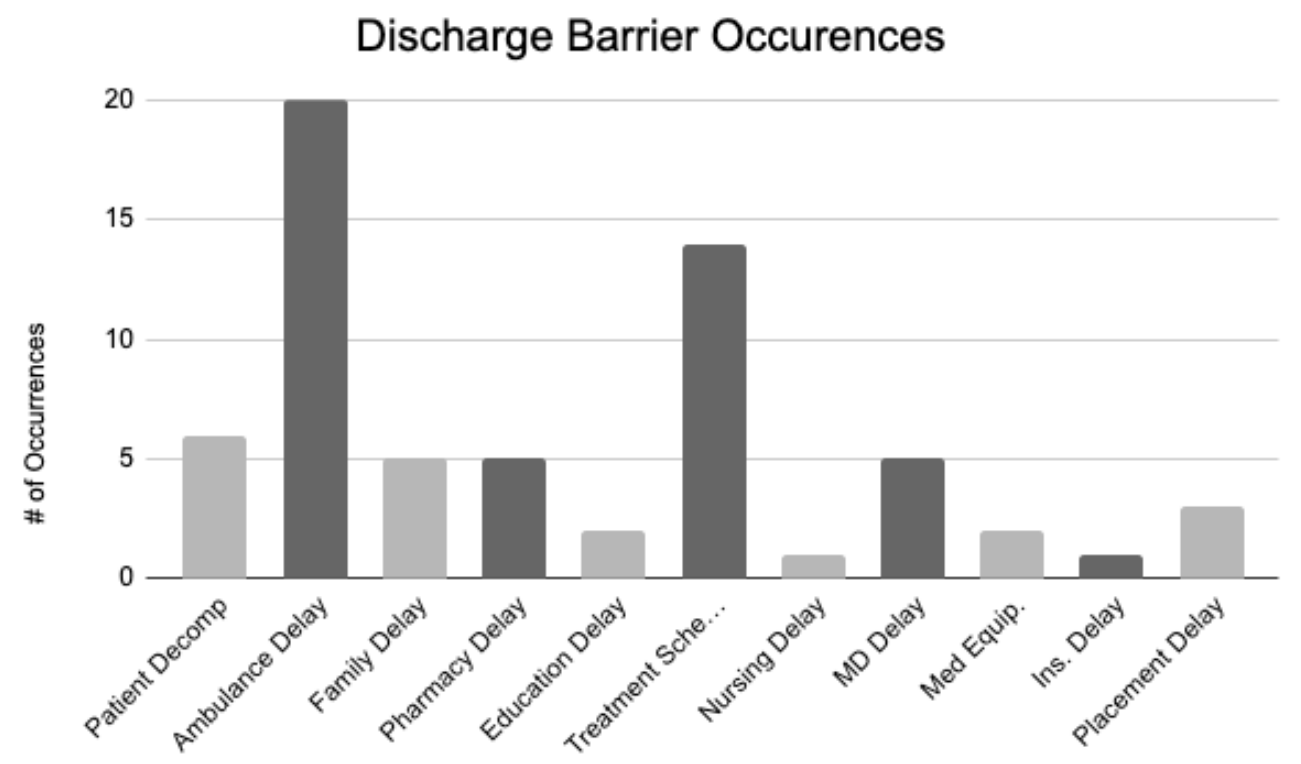

Figure 1. Discharge barrier occurrence count provided by bedside nurse and case management communication

M20 patients did not have 10 a.m. orders because patients: 1) were not seen early in the morning before rounds, 2) there was a lack of communication between team members, or 3) the patients decompensated and were no longer eligible for discharge. The primary reasons why beds with an order before $10 \mathrm{a} . \mathrm{m}$. were not selected for the M20 list the day before included: 1) lack of knowledge about the list, 2) not being informed of discharges until the day of, 3) transportation changes, and 4) patients with questionable discharge eligibility status.

\section{Discussion}

Previous studies show that discharge delays could adversely affect patient care and overall flow within a hospital. The practicum project was tested only within specific emergency medicine teams at one hospital, but the process and findings may be generalized to other institutions. Delays and barriers are inevitable within the discharge process. Primary barriers are sometimes unavoidable and out of the direct control of patients and staff. When presenting the findings to the discharge affinity group, the student investigators encouraged scheduling transportation as soon as possible, updating case management and nurses on M20 list usage, and scheduling treatments in advance when possible.

In the future, the affinity group will use this project's results to better understand barriers and discrepancies within the discharge process. The most important takeaway is how vital communication and planning are for discharge timeliness and overall performance. Procedures set in place will not be carried out efficiently without proper communication between every stakeholder involved. There were multiple staff misunderstandings involving tools like the 
M20 list and deeming a patient medically ready. These classifications and processes should be standardized as much as possible to ensure discharge efficiency. When feasible, it is essential to plan and communicate to facilitate discharge timeliness.

Additionally, the value of the undergraduate contribution to healthcare quality improvement projects is demonstrated through this project as data collection needed to be conducted in real-time. The primary barriers to discharge timeliness were found to be ambulance delays and scheduled treatments. To ensure minimal delays, patient care teams should identify patients ready for discharge and perform or schedule discharge tasks as early as possible.

\section{Limitations}

This project has a few limitations. Limitations included: collection availability, small sample size, access to data retrieval systems, and data sources. Study investigators were only available to collect data on specific days as data collection was done in real-time. Thus, the data collected represented a small sample of the number of barriers experienced in the project's time frame. Additionally, only one investigator had access to SCM, making it more difficult to retrieve all of the information needed to contact and interview registered nurses and case managers. Finally, the primary source of data was qualitative interviews with hospital staff using Voalte communications. Coding and transforming these interviews into quantitative data for analysis may have led to discrepancies in categorization.

\section{Acknowledgments}

The authors would like to thank the University of Kentucky HealthCare for allowing us to participate in this improvement project, especially during the COVID-19 pandemic. Additionally, this submission would not be possible without the Clinical Leadership and Management program's practicum opportunities in the College of Health Sciences. Special thanks to Dr. Preetham Talari and Dr. Karen Clancy for guiding the authors along the way.

\section{References}

Okoniewska, B., Santana, M. J., Groshaus, H., Stajkovic, S., Cowles, J., Chakrovorty, D., \& Ghali, W. A. (2015). Barriers to discharge in an acute care medical teaching unit: a qualitative analysis of health providers' perceptions. Journal of multidisciplinary healthcare, 8, 83. https://doi.org/10.2147/JMDH.S72633

Ragavan, M. V., Svec, D., \& Shieh, L. (2017). Barriers to timely discharge from the general medicine service at an academic teaching hospital. Postgraduate Medical Journal, 93(1103), 528-533. https://pmj.bmj.com/content/93/1103/528

Rohatgi, N., Kane, M., Winget, M., Haji-Sheikhi, F., \& Ahuja, N. (2018). Factors associated with delayed discharge on general medicine service at an academic medical center. The Journal for Healthcare Quality (JHQ), 40(6), 329335. https://doi.org/10.1097/JHQ.0000000000000126

Rojas-García, A., Turner, S., Pizzo, E., Hudson, E., Thomas, J., \& Raine, R. (2018). Impact and experiences of delayed discharge: A mixed-studies systematic review. Health Expectations, 21(1), 41-56.

https://doi.org/10.1111/hex.12619 